\title{
TGF- $\beta$ is a critical mediator of acute lung injury
}

\author{
Jean-Francois Pittet, ${ }^{1}$ Mark J.D. Griffiths, ${ }^{2}$ Tom Geiser ${ }^{3}$ Naftali Kaminski, ${ }^{2}$ \\ Stephen L. Dalton, ${ }^{2}$ Xiaozhu Huang, ${ }^{2}$ Lou Anne S. Brown, ${ }^{4}$ Phillip J. Gotwals, ${ }^{5}$ \\ Victor E. Koteliansky, ${ }^{5}$ Michael A. Matthay, ${ }^{6}$ and Dean Sheppard ${ }^{2}$
}

\author{
${ }^{1}$ Departments of Anesthesia and Surgery, \\ ${ }^{2}$ Department of Medicine and the Lung Biology Center, and \\ ${ }^{3}$ Cardiovascular Research Institute, University of California San Francisco, San Francisco, California, USA \\ ${ }^{4}$ Department of Pediatrics, Emory University, Atlanta, Georgia, USA \\ ${ }^{5}$ USA Biogen Inc., Cambridge, Massachusetts, USA \\ ${ }^{6}$ Department of Medicine and the Cardiovascular Research Institute, University of California San Francisco, \\ San Francisco, California, USA
}

Address all correspondence to: Dean Sheppard, Lung Biology Center, Box 0854, University of California San Francisco, San Francisco, California 94110, USA. Phone: (415) 206-5901; Fax: (415) 206-4123; E-mail: deans@itsa.ucsf.edu.

Received for publication December 8, 2000, and accepted in revised form May 8, 2001.

\begin{abstract}
We have shown that the integrin $\alpha v \beta 6$ activates latent TGF- $\beta$ in the lungs and skin. We show here that mice lacking this integrin are completely protected from pulmonary edema in a model of bleomycininduced acute lung injury (ALI). Pharmacologic inhibition of TGF- $\beta$ also protected wild-type mice from pulmonary edema induced by bleomycin or Escherichia coli endotoxin. TGF- $\beta$ directly increased alveolar epithelial permeability in vitro by a mechanism that involved depletion of intracellular glutathione. These data suggest that integrin-mediated local activation of TGF- $\beta$ is critical to the development of pulmonary edema in ALI and that blocking TGF- $\beta$ or its activation could be effective treatments for this currently untreatable disorder.
\end{abstract}

J. Clin. Invest. 107:1537-1544 (2001).

\section{Introduction}

Acute lung injury (ALI) is a devastating syndrome with an overall mortality rate of $30-40 \%$ (1). The syndrome is characterized by flooding of alveolar spaces with a protein-rich exudate that impairs pulmonary gas exchange leading to arterial hypoxemia and respiratory failure. Although this syndrome can follow lung injury and inflammation from multiple causes (e.g., sepsis, pancreatitis, hemorrhagic shock, or toxic inhalation), the molecular steps regulating the development of alveolar flooding are poorly understood, and no specific pharmacologic therapies are currently available. In mice, ALI can be modeled by administration of a single dose of intratracheal bleomycin, a drug that can also cause a similar syndrome in humans $(2,3)$.

The cytokine TGF- $\beta$ plays a critical role in the resolution of tissue injury in multiple organs, including the lung (4). Following ALI, TGF- $\beta$ has been most thoroughly evaluated during the late phases of tissue repair, where it plays a critical role in the development of pulmonary fibrosis $(5,6)$. However, in a recent study evaluating global patterns of gene expression following bleomycin-induced lung injury, we found that expression levels of several TGF- $\beta$-inducible genes were dramatically increased as early as 2 days after the induction of injury (7), a time point that precedes the maximal increase in alveolar flooding. Furthermore, in patients the TGF- $\beta$-inducible gene, procollagen III, is one of the earliest predictors of the severity of ALI $(8,9)$. In vitro TGF- $\beta$ has been shown to directly increase the permeability of endothelial monolayers (10). We therefore reasoned that TGF- $\beta$ might be active early in ALI and could potentially contribute to the development of the pulmonary edema that characterizes this disorder.

As a first step to assess the possible role of TGF- $\beta$ in ALI, we compared the increases in lung permeability after treatment with intratracheal bleomycin in wildtype mice and in mice lacking the epithelial integrin $\alpha v \beta 6$, an integrin that we have shown plays a critical role in activation of latent TGF- $\beta$ in the lungs and skin (11). Wild-type mice developed a significant increase in lung permeability to water and protein after bleomycin, but there was no significant change in either index of pulmonary edema in mice deficient in the $\alpha v \beta 6$ integrin. To more directly assess the role of TGF- $\beta$ in this process, we administered a soluble TGF- $\beta$ inhibitor (a TGF- $\beta$ type II receptor-IgG-Fc chimera) to wild-type mice treated with bleomycin or Escherichia coli endotoxin. Administration of this soluble receptor has been shown previously to inhibit several TGF- $\beta$-mediated responses, in vitro and in vivo $(12,13)$. Pharmacologic inhibition of TGF- $\beta$ protected wild-type mice from pulmonary edema. Finally, we examined whether active TGF- $\beta$ would directly disrupt the integrity of the alveolar epithelial barrier. TGF- $\beta$ directly increased alveolar epithelial permeability in vitro by a mechanism that involved depletion of intracellular glutathione. These data suggest that TGF- $\beta$, locally activated by the $\alpha v \beta 6$ integrin, is 
a critical mediator of pulmonary edema in ALI. Blocking TGF- $\beta$ or its activation could thus be effective treatments for this currently untreatable disorder.

\section{Methods}

Mice. Wild-type mice, 129/terSVEMS, were obtained from The Jackson Laboratories (Bar Harbor, Maine, USA). $\beta 6^{-/-}$ mice were bred and maintained according to institutional, state, and federal guidelines. Age- and sex-matched 8to 12-week-old littermates were used in all experiments. Intratracheal bleomycin or E. coli endotoxin administration. Wild-type or $\beta 6^{-/-}$mice were given intratracheal saline or bleomycin (0.05 IU in $60 \mu \mathrm{l}$ sterile saline; Meade Johnson, Princeton, New Jersey, USA). Five days later, lung permeability was measured 4 hours after intraperitoneal injection of $0.5 \mu \mathrm{Ci}$ of ${ }^{125} \mathrm{I}$-albumin. As an index of lung edema we measured lung wet/dry weight ratios gravimetrically using standard methods, as in our previous studies (14). Briefly, before exsanguination, a blood sample was obtained to measure the hemoglobin concentration and the water-to-dry weight ratio of blood for the extravascular lung water calculation. Lungs were homogenized and the extravascular lung water determined by calculating the water-to-dry weight ratio using the following equation: $W / D=$ $Q_{\text {wet }} / Q_{\text {dry }}$ in which $Q_{\text {wet }}$ is the difference between the water content of the lung homogenate and the water content of the blood in the lung and $Q_{\text {dry }}$ is the dry lung weight calculated as the weight of the lungs minus the blood and water volumes in the lung. As an index of lung permeability we calculated the ratio of accumulated tracer (counts per minute per gram of lung tissue) in the extravascular spaces of the lungs (calculated by the gravimetric method) to the mean plasma value (counts per minute per gram of plasma).

In a second series of experiments, soluble chimeric TGF- $\beta$ type II receptor $(25 \mu$ g diluted in $100 \mu \mathrm{l}$ sterile saline; USA Biogen Inc., Cambridge, Massachusetts, USA) or saline alone was administered intravenously just before and 48 and 96 hours after bleomycin instillation (pretreatment group) or 24 and 72 hours after bleomycin instillation (treatment group). The third series of experiments was designed to determine whether pharmacological inhibition of TGF- $\beta 1$ with the soluble chimeric TGF- $\beta$ type II receptor would protect against development of ALI in a more clinically relevant model of ALI. Wild-type mice were pretreated with the soluble chimeric TGF- $\beta$ type II receptor or its vehicle just before airspace instillation of E. coli endotoxin or of its vehicle (L-2880; $50 \mu \mathrm{g}$ diluted in $50 \mu \mathrm{l}$ sterile saline) (Sigma Chemical Co., St. Louis, Missouri, USA). Indices of lung permeability to protein and of pulmonary edema were measured as described above.

To determine that the soluble receptor is a potent inhibitor of both exogenous recombinant TGF- $\beta$ and of $\alpha v \beta 6$-mediated activation of TGF- $\beta, 1.6 \times 10^{4} \mathrm{mink}$ lung epithelial reporter cells (TMLC cells; ref. 15), expressing firefly luciferase under the control of a portion of the TGF- $\beta$-sensitive plasminogen activator inhibitor-1 promoter, were suspended in DMEM containing 10\% FCS, plated in microtiter wells, and allowed to attach for 1 hour. Test solutions (rhTGF- $\beta 1$ and/or soluble TGF- $\beta$ receptor) and/or test cells ( $\beta 6$ transfected SW480 cells plated at the same density) were added for 16-20 hours, and lysates were assayed for luciferase activity as described (15). In all experiments, one-way ANOVA and Fisher's exact $t$ test were used to compare experimental with control groups.

Bronchoalveolar lavage and flow cytometry. Mice were killed by cervical dislocation, and a blunt needle was inserted into the upper trachea. Lavage was performed by introducing five sequentia, $0.8-\mathrm{ml}$ aliquots of PBS into the lungs and carefully withdrawing the fluid. Bronchoalveolar lavage (BAL) fluid was centrifuged at $130 \mathrm{~g}$ for 5 minutes and the cell pellets were resuspended in 1-ml red blood cell lysis buffer (Sigma Chemical Co.). Total cells were counted in a hemocytometer, and a cytospin slide preparation was made from a aliquot of each sample. Slides were stained with a Diff-Quick stain set (Dade Diagnostic of Puerto Rico Inc., Aguada, Puerto Rico), and a differential cell count of 300 cells was made.

The remaining BAL cells were blocked with normal goat serum (Vector Laboratories, Burlingame, California, USA) at $4^{\circ} \mathrm{C}$ for 10 minutes and then labeled with a $\mathrm{mAb}$ against $\mathrm{MHC}$ class II for 20 minutes at $4^{\circ} \mathrm{C}$. After washing twice with PBS, stained cells were resuspended in $100 \mu \mathrm{l}$ of PBS and analyzed by flow cytometry on a Becton Dickinson Immunocytometry Systems FACSort (San Jose, California, USA).

Histology. The trachea and both lungs were fixed by inflation at $25 \mathrm{~cm} \mathrm{H}_{2} \mathrm{O}$ with $10 \%$ formalin and embedded in paraffin for histology. Five-micrometer sections were fixed in Histochoice (Fisher Scientific Co., Pittsburgh, Pennsylvania, USA) for staining with hematoxylin and eosin.

Transepithelial electrical resistance measurements. Rat ATII cells were isolated by elastase digestion and selective adhesion plating on rat immunoglobulin G (16). Cells were plated at a concentration of $10^{6}$ cells $/ \mathrm{cm}^{2}$ in DMEM-H21 medium with $10 \%$ FCS. Twenty-four hours later, ATII cell monolayers were exposed to $10-400 \mathrm{pM}$ rhTGF- $\beta 1$ for 24 hours or to $80 \mathrm{pM}$ for 6-24 hours. Transepithelial electrical resistance (TER) was measured at room temperature with an epithelial voltmeter (Millicell-ERS; Millipore Corp., Bedford, Massachusetts, USA) using Ag-AgCl electrodes. Before measurements, electrodes were sterilized in $70 \%$ ethanol for 10 minutes and allowed to equilibrate in the cell culture medium for 60 minutes.

In a second series of experiments, ATII cell monolayers were exposed 24 hours after plating to active rhTGF- $\beta 1(80 \mathrm{pM})$ or to its vehicle in the presence or absence of either a mouse $\mathrm{mAb}$ against all isoforms of TGF- $\beta$ (1D11; R\&D Systems Inc., Minneapolis, Minnesota, USA) or an isotypic irrelevant Ab. Twenty-four hours later, TER was measured as described above. In a third series of experiments, ATII cell 


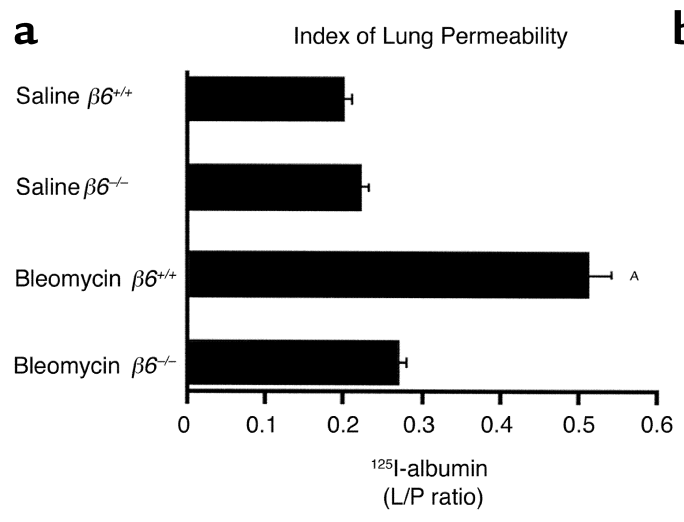

b

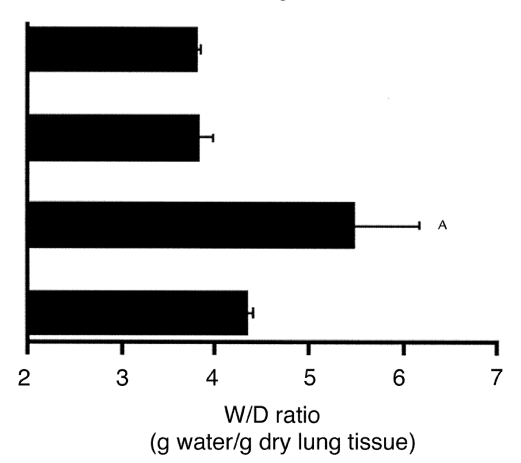

\section{Figure 1}

$\beta 6^{-/-}$mice are protected against bleomycin-induced pulmonary edema. Lung permeability to protein (L/P, lung/plasma ratio) (a) and extravascular water content of the lung $(W / D$, wet/dry ratio $)(\mathbf{b})$ in wild-type $\left(\beta 6^{+++}\right)$and $\beta 6^{-/-}$mice 5 days after treatment with intratracheal saline or bleomycin. Data (means \pm SEM) are representative of at least two comparable experiments with five mice per group; ${ }^{A} P<$ 0.05 relative to saline-treated mice and bleomycin treated $\beta 6^{-/-}$mice. monolayers were exposed 24 hours after plating to active rhTGF- $\beta 1(80 \mathrm{pM})$ or to its vehicle in the presence of either a monoethyl ester of glutathione (100-400 pM; Sigma Chemical Co.) or its vehicle. Twenty-four hours later, TER was measured as described above. Reduced and oxidized glutathione were measured using a variation of the HPLC method reported by Martin (17).

In a fourth series of experiments, ATII cell monolayers were exposed 24 hours after plating to active rhTGF- $\beta 1(80 \mathrm{pM})$ or its vehicle in the presence of either ML-7 $(3 \mu \mathrm{M})$ or ML-9 $(50 \mu \mathrm{M})$, specific inhibitors of the myosin light chain kinase or their vehicles. Twenty-four hours later, TER was measured as described above. In all experiments, one-way ANOVA and Fisher's exact $t$ test were used to compare experimental groups with control groups.

\section{Results}

$\beta 6^{-/-}$mice are protected against bleomycin-induced pulmonary edema. We have reported recently that cells expressing the $\alpha v \beta 6$ integrin activate TGF- $\beta 1$ and that mice lacking this integrin are protected from pulmonary fibrosis, a late response to lung injury that is critically dependent on TGF- $\beta$ activity (11). To determine whether locally activated TGF- $\beta$ modulates the alveolar flooding that causes morbidity and mortality early after lung injury, we compared the increases in lung permeability 5 days after treatment with intratracheal bleomycin in wild-type mice and in mice lacking the epithelial integrin $\alpha v \beta 6$. We chose this time point because in preliminary studies we found that 129/terSVEMS mice develop maximal increases in lung permeability within 5 days after treatment with bleomycin. Intratracheal bleomycin (0.05 IU) induced the development of protein-rich pulmonary edema in $\beta 6^{+/+}$but not in $\beta 6^{-/-}$mice. Compared with saline-treated mice, there was a significant increase in the lung permeability to protein (Figure 1a) and in the extravascular water content of the lung (Figure 1b) in bleomycin-treated $\beta 6^{+/+}$mice compared with salinetreated mice. In contrast, there was no change in either index of pulmonary edema in bleomycin-treated $\beta 6^{-/-}$ mice (Figure 1, a and b). In preliminary studies, there was no increase in pulmonary edema at any time point up to 15 days after intratracheal bleomycin in $\beta 6^{-/-}$ mice (data not shown).

BAL fluid cellularity of $\beta 6^{-/-}$mice was characterized at baseline by lymphocytic airway inflammation and macrophage activation (foamy macrophages) compared with their wild-type littermates, as we have reported previously (18) (Figure 2). Five days after instillation of bleomycin, there was an exaggeration of the airway inflammatory response in $\beta 6^{-/-}$mice compared with $\beta 6^{+/+}$wild-type mice. Bleomycin increased the numbers of lymphocytes, neutrophils,

\section{Figure 2}

Effect of airspace instillation of bleomycin on differential cell count and macrophage expression of MHC II in BAL fluid in $\beta 6^{+/+}$and $\beta 6^{-/-}$mice. Means $( \pm$ SEM) of differential cell count obtained from BAL of each of six mice in both experimental groups. Values for MHC II expression on macrophages were obtained by flow cytometry.

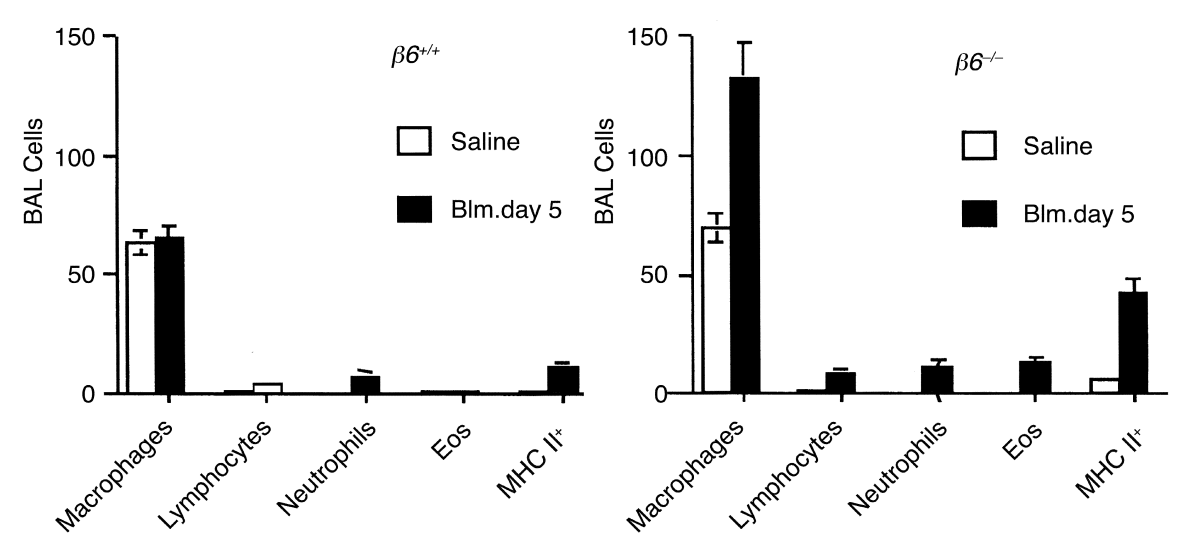


a
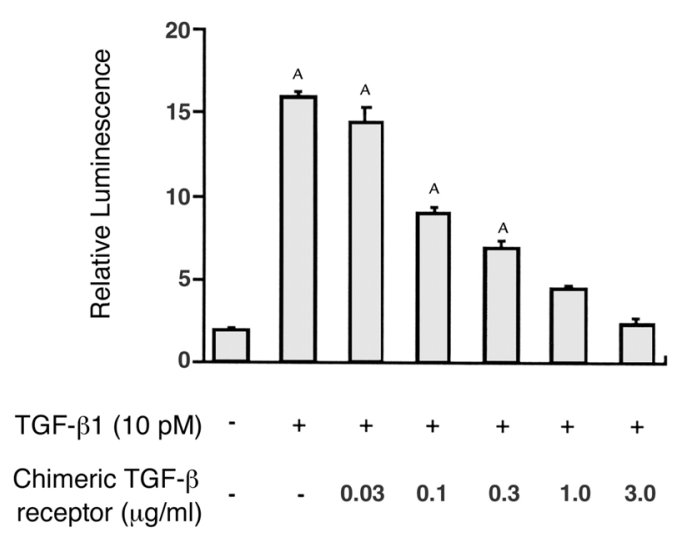

b
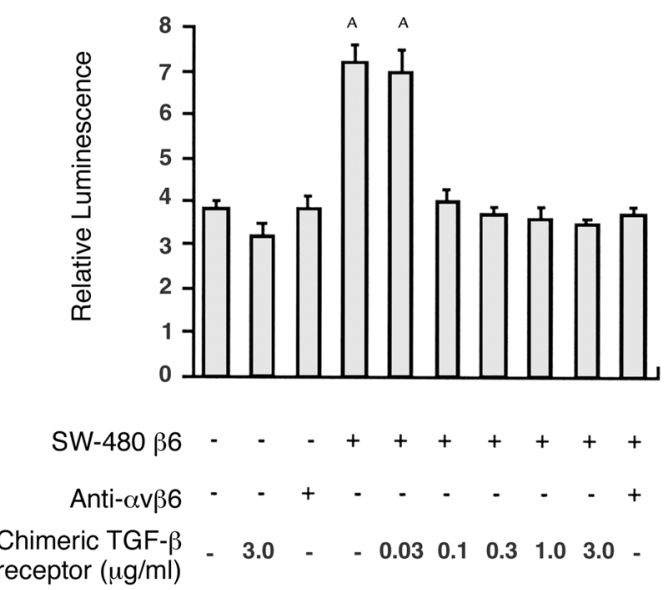

c

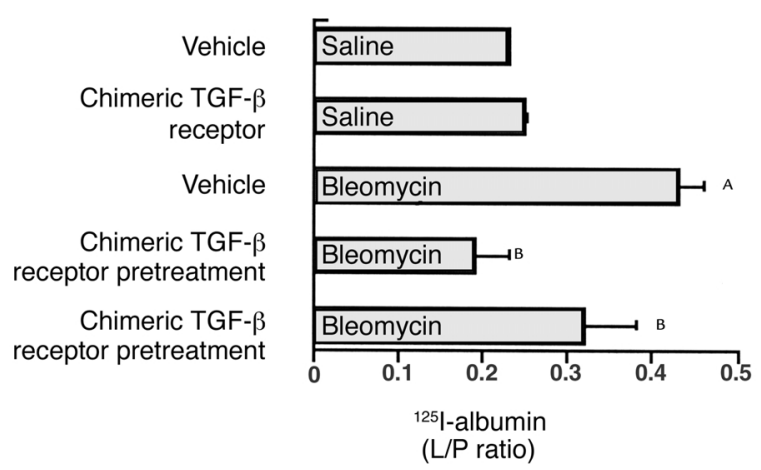

Figure 3

Soluble chimeric TGF- $\beta$ type II receptor inhibits TGF- $\beta 1$ activity in $\beta 6$-expressing cells and protects $\beta 6^{+/+}$mice against bleomycininduced pulmonary edema. TGF- $\beta$ activity is measured as relative luminescence of TMLC cells stably expressing a portion of the plasminogen activator inhibitor-1 promoter (12) in response to exogenous rhTGF- $\beta 1$ (a) or $\beta 6$-transfected SW480 cells (b) in the presence of increasing concentrations of TGF- $\beta$ type II receptor-IgG-Fc chimera. Means $( \pm S E M)$ of three experiments done in duplicate are shown. (c) Lung protein permeability in wild-type mice treated with saline or bleomycin and given either TGF- $\beta$ type II receptor chimera or saline every 48 hours beginning either just before (pretreatment) or 24 hours after (treatment) intratracheal instillation. Data (means \pm SEM) are representative of at least two comparable experiments with five mice per group; ${ }^{A} P<0.05$ relative to saline-treated mice; ${ }^{\mathrm{B}} P<0.05$ relative to bleomycin-treated mice that received vehicle. and MHC II-expressing macrophages in the airspaces of wild-type mice, but all of these effects were greater in the airspaces of $\beta 6^{-/-}$mice. Interestingly, changes in BAL cellularity in $\beta 6^{-/-}$mice did not correlate with changes in lung protein and water permeability after instillation of bleomycin.

Soluble chimeric TGF- $\beta$ type II receptor inhibits TGF- $\beta 1$ activity in $\beta 6$-expressing cells and protects $\beta 6^{+/+}$mice against bleomycin-induced pulmonary edema. To more directly assess the role of TGF- $\beta$ in this process, we used a soluble TGF- $\beta$ type II receptorIgG-Fc chimera to inhibit the binding of active TGF- $\beta$ to its receptor. We first determined that this soluble TGF- $\beta$ receptor is a potent inhibitor of both exogenous recombinant TGF- $\beta$ and of $\alpha v \beta 6$-mediated activation of TGF- $\beta$. Increasing concentrations of the TGF- $\beta$ type II receptor-IgG-Fc chimera caused a progressive decrease in the luciferase activity resulting from the stimulation of TMLC cells stably expressing a portion of the plasminogen activator inhibitor-1 promoter with active rhTGF- $\beta 1$ (Figure $3 a$ ). The soluble TGF- $\beta$ type II receptor-IgG-Fc chimera also caused concentrationdependent inhibition of TGF- $\beta 1$ activity in a coculture bioassay containing TMLC and $\beta 6$-transfected SW480 cells (Figure $3 b$ ). Soluble TGF- $\beta$ type II receptorIgG-Fc chimera or saline alone was administered intravenously just before and 48 and 96 hours after bleomycin instillation (pretreatment group) or 24 and 72 hours after bleomycin instillation (treatment group). Pretreatment of wild-type mice with a soluble TGF- $\beta$ type II receptor-IgG-Fc chimera completely prevented the increase in lung protein permeability observed during the acute phase of lung injury after airspace instillation of bleomycin (Figure 3c). Significant protection against alveolar edema was still observed when administration of this soluble chimeric TGF- $\beta$ receptor was started 24 hours after the challenge with bleomycin (Figure 3c). We have reported previously that the amount of total TGF- $\beta 1$ was not different between mice lacking $\alpha v \beta 6$ integrin and their wild-type littermates and was not significantly affected by treatment with bleomycin (11).

Morphologic evidence of bleomycin-induced pulmonary edema is absent in $\beta 6^{+/+}$mice and in wild-type mice treated with TGF-II receptor chimera. To further examine the significance of the measurements of lung water and permeability described above, we also evaluated lung morphology in wild-type and $\beta 6^{+/+}$mice after treatment with bleomycin or saline and in wildtype mice treated with the TGF- $\beta$ type II receptor chimera. In wild-type mice instilled with bleomycin, there was accumulation of red cells and proteinaceous material in the alveoli (Figure 4). This was observed neither in $\alpha v \beta 6$ knockout mice nor in wild-type mice that were pretreated with a soluble chimeric TGF- $\beta$ type II receptor before exposure to bleomycin (Figure 4). These data thus indicate that bleomycin caused injury to both the lung endothelial and the alveolar epithelial barriers. 

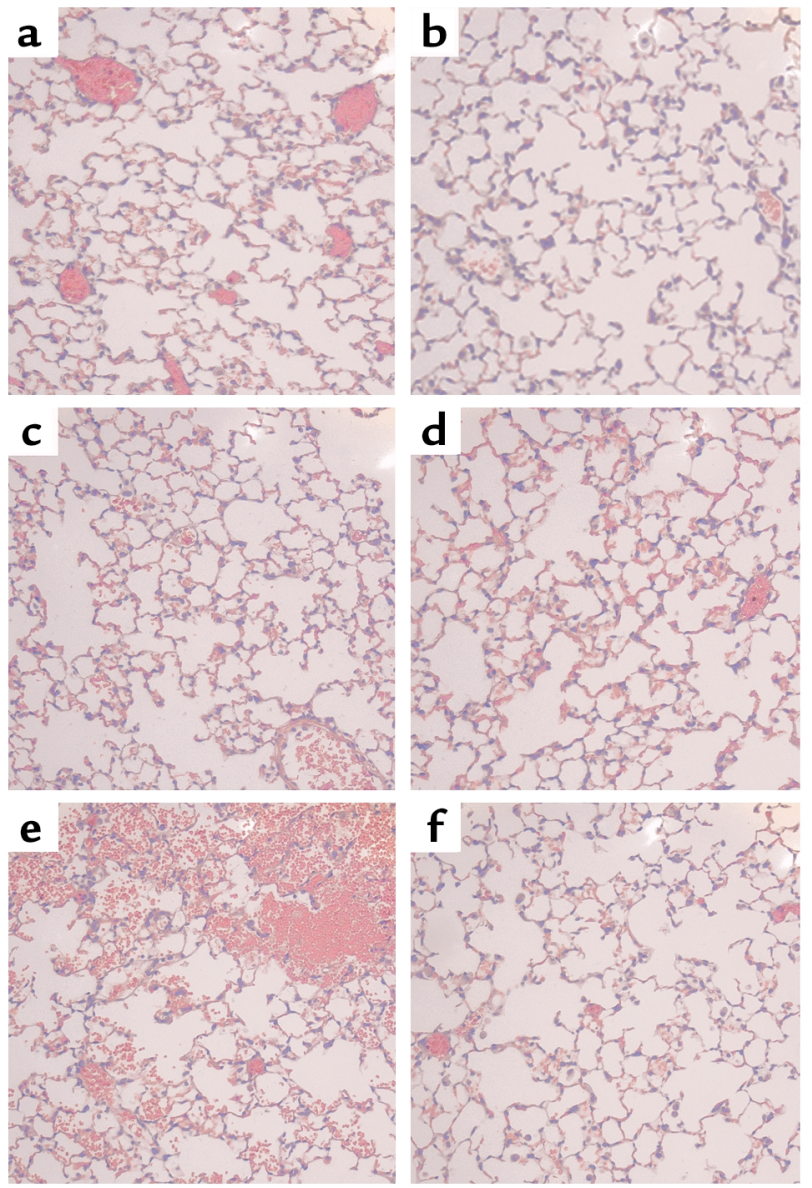

Figure 4

Lung histology. Low-power photomicrographs of lung sections stained with hematoxylin and eosin. (a) $\beta 6^{-/-}$mouse instilled with PBS shows a normal lung morphology. (b) $\beta 6^{-/-}$mouse instilled with bleomycin does not show any red blood cell leak or accumulation of proteinaceous material into its airspaces. (c and $\mathbf{d}$ ) $\beta 6^{+/+}$mice instilled with PBS and injected either with the soluble chimeric TGF- $\beta$ type II receptor (c) or its vehicle (d) have a normal lung morphology. (e) Accumulation of red blood cells and proteinaceous material in the alveolar spaces of a $\beta 6^{+/+}$mouse instilled with bleomycin and injected with the vehicle for the soluble chimeric TGF- $\beta$ type II receptor. (f) Absence of red blood cells in the airspaces of a $\beta 6^{+/+}$mouse instilled with bleomycin and pretreated with the soluble chimeric TGF- $\beta$ type II receptor.

Blockade of TGF- $\beta$ protects wild-type mice against endotoxin-induced pulmonary edema. We next determined whether these findings would apply to another model of ALI, airspace instillation of $E$. coli endotoxin, which is a more classic initiator of ALI than instillation of bleomycin. We found that the instillation of E. coli endotoxin $(50 \mu \mathrm{g}$ in $50 \mu \mathrm{l}$ ) into the airspaces of wild-type mice caused a significant increase in protein permeability and extravascular water content of the lung (Figure 5). This effect was significantly attenuated by the intravenous administration of the chimeric TGF- $\beta$ receptor (Figure 5). These findings and our present results are therefore consistent with a model of lung injury in which tissue injury induces expression and/or activation of the $\alpha v \beta 6$ integrin, which in turn locally activates TGF- $\beta 1$ already present in latent form in the lung.
rbTGF- $\beta 1$ decreases the TER across primary cultures of rat alveolar epithelial type II cell monolayers by decreasing their intracellular content of reduced glutathione. Two separate barriers form the alveolar-capillary barrier, the microvascular endothelium and the alveolar epithelium. The acute phase of ALI often involves dysfunction of both barriers of the lung. However, the alveolar epithelium is much less permeable than the lung endothelium under physiological conditions. The loss of alveolar epithelial integrity has numerous consequences for patients with ALI. It contributes to alveolar flooding, disrupts secretion of surfactant, impairs removal of edema fluid from the alveolar space, and strongly affects patient survival. To assess whether active TGF- $\beta 1$ would directly disrupt the integrity of the alveolar epithelial barrier, primary cultures of rat alveolar epithelial type II (ATII) cells were incubated with increasing concentrations of active rhTGF- $\beta 1$. Active rhTGF- $\beta 1$ caused a dose- and time-dependent decrease in TER (Figure 6a). This TGF- $\beta 1$-dependent loss of the integrity of the alveolar epithelial barrier was prevented by pretreatment with a mouse $\mathrm{mAb}$ to TGF- $\beta 1$, but not by pretreatment with an irrelevant isotypic $\mathrm{Ab}$ (Figure 6b), and was not associated with an increase in the rate of apoptosis or necrosis in these monolayers (as determined by TUNEL staining and trypan-blue exclusion; data not shown).

We have recently reported that glutathione (GSH) depletion markedly increased the permeability of the alveolar epithelium to protein (19), and Arsalane and coworkers have shown that TGF- $\beta$ is a potent inhibitor of GSH synthesis in lung epithelial cells (20). We therefore sought to determine whether the effects of TGF- $\beta$ on epithelial permeability were mediated by loss of GSH. The rhTGF- $\beta 1$-dependent decrease in TER was progressively attenuated by pretreatment with increas-

Index of Lung Permeability

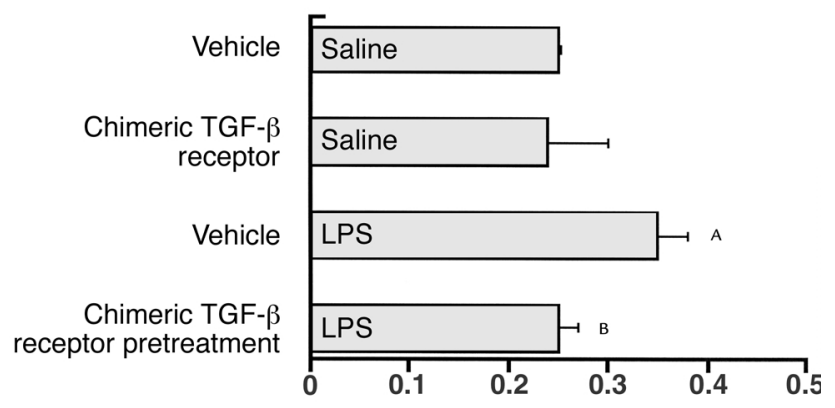

\section{Figure 5}

Soluble chimeric TGF- $\beta$ type II receptor protects $\beta 6^{+/+}$mice against E. coli endotoxin-induced pulmonary edema. Lung protein permeability in wild-type mice treated with saline or E. coli endotoxin and given either TGF- $\beta$ type II receptor chimera or saline just before intratracheal instillation. Data (means \pm SEM) are representative of two comparable experiments with five mice per group; ${ }^{A} P<0.05$ relative to saline-treated mice; ${ }^{\mathrm{B}} P<0.05$ relative to bleomycin-treated mice that received vehicle. 
a

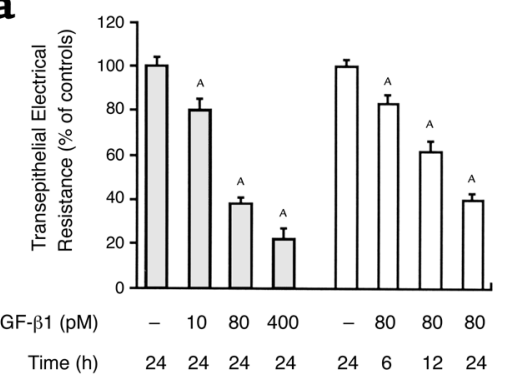

b

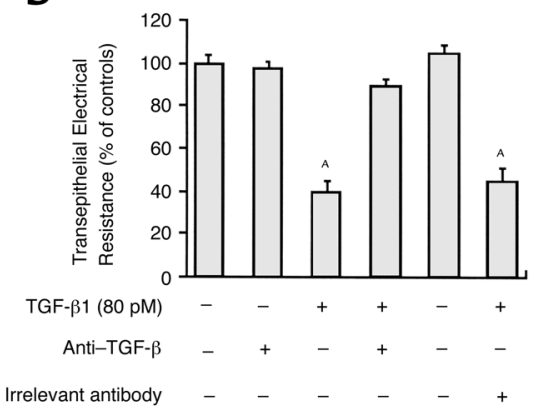

C

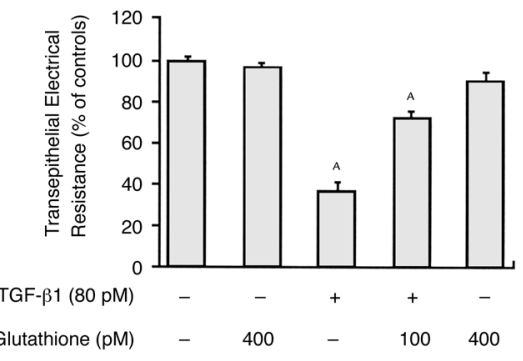

d

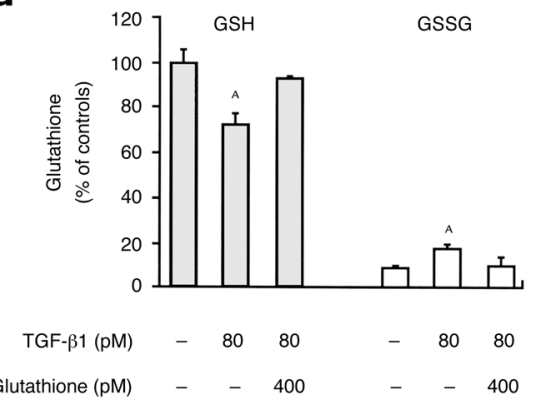

e

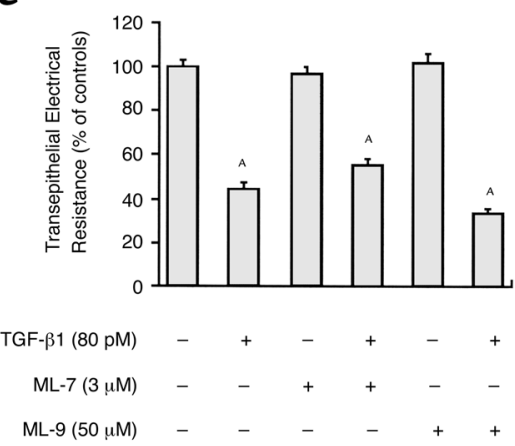

\section{Figure 6}

rhTGF- $\beta 1$ decreases the TER across primary cultures of rat ATII cell monolayers by decreasing their intracellular content of reduced GSH. Transepithelial resistance of rat ATII monolayers treated with rhTGF- $\beta 1$ alone (a) or in the presence of either anti-TGF- $\beta$ Ab (b) or exogenous GSH (c). (d) Effects of rhTGF- $\beta 1$ on ATII cell content of GSH and GSSG. (e) Effects of inhibitors of the myosin light chain kinase on the TGF- $\beta$-induced decrease in TER. Means $( \pm$ SEM) of three experiments done in duplicate are shown for each panel. For all experiments, AP $<0.05$ relative to vehicle-treated ATII cell monolayers.

ing doses of a GSH monoethyl ester (Figure 6c). In addition, active rhTGF- $\beta 1$ caused a significant decrease in the ATII cell content of reduced GSH associated with a small increase in its intracellular oxidized form (GSSG) (Figure 6d). Interestingly, in contrast to the results reported previously for the endothelial barrier $(10)$, the TGF- $\beta$-induced increase in alveolar epithelial permeability was not dependent on the activation of myosin light-chain kinase. Indeed, two unrelated inhibitors of this enzyme ML-7 and ML-9, did not prevent the rhTGF- $\beta 1$-dependent TER decrease across primary cultures of rat ATII cell monolayers (Figure 6e).

\section{Discussion}

The results of this study demonstrate that mice lacking the integrin $\alpha v \beta 6$, an integrin we have shown previously is capable of locally activating latent TGF- $\beta(11)$, are dramatically protected from pulmonary edema in a bleomycin-induced model of ALI. Protection of $\beta 6^{-/-}$ mice was not explained by a difference in the alveolar inflammatory response to bleomycin between mice deficient in $\alpha v \beta 6$ integrin and their wild-type littermates, since, as we have reported previously (11) and have shown again in the present study (Figure 2), the inflammatory response to bleomycin is actually enhanced in $\beta 6$ subunit knockout mice. In addition to inducing TGF- $\beta$ activation, $\alpha v \beta 6$ plays a role in cell proliferation and migration $(21,22)$ and could thus have been affecting the development of pulmonary edema by mechanisms independent of TGF- $\beta$. Furthermore, the baseline inflammation present in the lungs of $\beta 6$ subunit knockout mice could have conditioned the lungs indirectly to resist subsequent injury. However, administration of a TGF- $\beta$ antagonist to wild-type mice also prevented the development of pulmonary edema in the same model of ALI, directly implicating TGF- $\beta$ in this process. Moreover, inhibition of TGF- $\beta$ significantly inhibited pulmonary edema when it was administered as late as 24 hours after the induction of bleomycin-mediated injury, suggesting that if a similar mechanism is involved in ALI in humans, blocking this pathway could be therapeutically useful. Comparable results were obtained with another model using endotoxin, a more classic initiator of ALI than instillation of bleomycin. We found that the instillation of $E$. coli endotoxin into the airspaces of wildtype littermates caused a significant increase in protein permeability and extravascular water content of the lung. This effect was also significantly attenuated by the intravenous administration of a TGF- $\beta$ antagonist (Figure 5). Taken together, these findings indicate that $\alpha v \beta 6$ integrin-mediated, spatially restricted activation of TGF- $\beta 1$ in the distal airspaces of the lung may play an important role in the development of pulmonary edema during the early phase of ALI.

As noted above, increased permeability of both the endothelium and epithelium contribute to alveolar 
flooding in ALI. TGF- $\beta 1$ could increase endothelial permeability through a previously reported effect of increasing gaps between endothelial cells (10). This effect appears to result from endothelial cell contraction through the activation of a myosin light chain kinase-dependent signaling cascade. However, TGF- $\beta$ 's in vitro effect on the integrity of vascular endothelium started within 1-2 hours, was maximal 8-9 hours after exposure to TGF- $\beta$, and appears to be dependent on the proliferative state and the degree of confluence of the endothelial cell monolayers. For example, quiescent cells have been reported to be resistant to this effect (23). Thus, it is uncertain whether effects of TGF- $\beta$ on the endothelium could entirely explain our in vivo results. In fact, lung morphology of wild-type mice instilled with bleomycin showed accumulation of red cells and proteinaceous material in the alveoli. This was observed neither in $\alpha v \beta 6$ knockout mice nor in wild-type mice that were pretreated with a soluble chimeric TGF- $\beta$ type II receptor before exposure to bleomycin (Figure 4). These data thus indicate that bleomycin caused injury to both the lung endothelial and the alveolar epithelial barriers. The alveolar epithelium is much less permeable than the lung endothelium under physiological conditions. As noted above, loss of alveolar epithelial integrity has numerous consequences for patients with ALI and strongly affects patient survival $(4,24)$. Our finding that rhTGF- $\beta 1$ caused a dose- and time-dependent increase in TER in vitro suggests that TGF- $\beta$ could directly increase the permeability of the alveolar epithelium, an effect that could be critical to its role in the induction of pulmonary edema in vivo.

The mechanism by which TGF- $\beta$ increases epithelial permeability appears to be distinct from the myosinlight chain kinase-dependent contraction reported for endothelial cells. The results of the current study suggest that TGF- $\beta$ increases epithelial permeability by a mechanism that depends on depletion of intracellular GSH and an increase in the concentration of its oxidized form, GSSG. The potential significance of these findings is supported by recent reports that GSH is depleted from epithelial lining fluid in experimental models of ALI (25) and in patients with ALI (26) and by our recent report that GSH depletion markedly increased the permeability of the alveolar epithelium to protein (19). A possible explanation for our findings is provided by evidence that TGF- $\beta$ inhibits GSH synthesis in lung epithelial cells by its transcriptional effect on the GSH rate-limiting enzyme $\gamma$-glutamylcysteine synthetase (20). Furthermore, TGF- $\beta$ has been shown to act as a pro-oxidant by increasing the cellular generation of $\mathrm{H}_{2} \mathrm{O}_{2}$ (27). Finally, GSSG itself has been shown recently to directly increase paracellular permeability in epithelial monolayers (28). However, one must be cautious in interpreting the in vitro experiments described here, since alterations in cellular oxidation/reduction could have a number of unmeasured effects in addition to the alterations in intracellular GSH/GSSG concentrations we describe.
TGF- $\beta$ is generally considered a central mediator of the resolution phase of tissue injury, inhibiting tissue inflammation and enhancing scarring. Our results, suggesting a critical role for this cytokine in the alveolar flooding that characterizes the early response to lung injury were therefore unexpected. However, a least one previous report did suggest a role for this cytokine early in a model of lung injury (29). In that study, an Ab to TGF- $\beta$ was shown to inhibit the induction of proinflammatory cytokines by alveolar macrophages after hemorrhagic shock. Since the authors did not report measurements of pulmonary edema in that study, its relevance to the current findings is uncertain.

The relevance of our results to ALI in humans remains an important question that cannot be completely addressed here because of the complexity of the syndrome and the inherent limitations of any murine model of ALI. However, our data suggest that activation of latent TGF- $\beta$ by interaction with the epithelial integrin $\alpha v \beta 6$ and subsequent direct effects of TGF- $\beta$ on alveolar epithelial cells (and perhaps adjacent endothelial cells) could play a critical role in the alveolar flooding that characterizes this often lethal syndrome. Mortality from ALI is related to the loss of epithelial barrier function (24) and to the production of collagen (8), an index of pulmonary fibrosis. The results of this and previous studies suggest that both of these determinants of mortality may be mediated by activated TGF- $\beta$. Current efforts to therapeutically intervene in this syndrome have been hampered by the fact that most interventions studied are effective only if administered before the onset of injury, while patients do not come to medical attention until after the lung injury has occurred. Since a TGF- $\beta$ inhibitor effectively attenuated alveolar flooding when administered as late as 24 hours after the onset of injury, targeting the integrin $\alpha v \beta 6$, the process of integrin-mediated TGF- $\beta$ activation or TGF- $\beta$ and its receptors could provide novel therapeutic strategies for treatment of this common, often fatal clinical syndrome.

\section{Acknowledgments}

This work was supported by NIH grants HL-51854 (to J.F. Pittet, M.A. Matthay), T32 HL-07185 (to M.J.D. Griffiths, N. Kaminski, X. Huang), and HL-47412, HL53949, and HL-33259 (to D. Sheppard).

\footnotetext{
1. Ware, L.B., and Matthay, M.A. 2000. The acute respiratory distress syndrome. N. Engl. J. Med. 342:1334-1349.

2. Hakkinen, P.J., et al. 1983. Potentiating effects of oxygen in lungs damaged by methylcyclopentadienyl manganese tricarbonyl, cadmium chloride, oleic acid, and antitumor drugs. Toxicol. Appl. Pharmacol. 67:55-69.

3. Van Barneveld, P.W., et al. 1987. Natural course of bleomycin-induced pneumonitis. A follow-up study. Am. Rev. Respir. Dis. 135:48-51.

4. Shull, M.M., et al. 1992. Targeted disruption of the mouse transforming growth factor-beta 1 gene results in multifocal inflammatory disease. Nature. 359:693-699.

5. Giri, S.N., Hyde, D.M., and Hollinger, M.A. 1993. Effect of antibody to transforming growth factor beta on bleomycin induced accumulation of lung collagen in mice. Thorax. 48:959-966.

6. Broekelmann, T., Limper, A.H., Colby, T.V., and MacDonald, J.A. 1991. Transforming growth factor beta 1 is present at sites of extracellular matrix gene expression in human pulmonary fibrosis. Proc. Natl. Acad. Sci. USA. 88:6642-6646.

7. Kaminski, N., et al. 2000. Global analysis of gene expression in pul-
} 
monary fibrosis reveals distinct programs regulating lung inflammation and fibrosis. Proc. Natl. Acad. Sci. USA. 97:1778-1783.

8. Chestnutt, A.N., Matthay, M.A., Tibayan, F.A., and Clark, J.G. 1997. Early detection of type III procollagen peptide in acute lung injury. Pathogenetic and prognostic significance. Am. J. Respir. Crit. Care Med. 156:840-845.

9. Pittet, J.F., Mackersie, R.C., Martin, T.R., and Matthay, M.A. 1997. Biological markers of acute lung injury: prognostic and pathogenetic significance. Am. J. Respir. Crit. Care Med. 155:1187-1205.

10. Hurst, V., Goldberg, P.L., Minnear, F.L., Heimark, R.L., and Vincent, P.A 1999. Rearrangement of adherens junctions by transforming growth factor-beta1: role of contraction. Am. J. Physiol. 276:L582-L595.

11. Munger, J.S., et al. 1999. The integrin alphavbeta 6 binds and activates latent TGF beta-1: a mechanism for regulating pulmonary inflammation and fibrosis. Cell. 96:319-328.

12. Wang, Q., et al. 1999. Reduction of bleomycin induced lung fibrosis by transforming growth factor beta soluble receptor in hamsters. Thorax. 54:805-812.

13. Smith, J.D., et al. 1999. Soluble transforming growth factor-beta type II receptor inhibits negative remodeling, fibroblast transdifferentiation, and intimal lesion formation but not endothelial growth. Circ. Res. 84:1212-1222.

14. Pittet, J.F., Wiener-Kronish, J.P., McElroy, M.C., Folkesson, H.G., and Matthay, M.A. 1994. Stimulation of alveolar epithelial liquid clearance by endogenous release of catecholamines in septic shock. J. Clin. Invest. 94:663-671.

15. Abe, M., et al. 1994. An assay for transforming growth factor-beta using cells transfected with a plasminogen activator inhibitor-1 promoterluciferase construct. Anal. Biochem. 216:276-284

16. Dobbs, L.G., Gonzalez, R., and Williams, M.C. 1986. An improved method for isolating type II cells in high yield and purity. Am. Rev. Respir. Dis. 134:141-145.

17. Martin, J., and White, I.M. 1991. Fluorimetric determination of oxidized and reduced glutathione in cells and tissues by high-performance liquid chromatography following derivatization with dansyl chloride. J. Chromatogr. 568:219-225.

18. Huang, X., et al. 1996. Inactivation of the integrin beta 6 subunit gene reveals a role of epithelial integrins in regulating inflammation in the lungs and skin. J. Cell Biol. 133:921-928.

19. Guidot, D.M., et al. 2000. Ethanol ingestion via glutathione depletion impairs alveolar epithelial barrier function in rats. Am. J. Physiol. 279:L127-L135.

20. Arsalane, K., et al. 1997. Transforming growth factor-beta1 is a potent inhibitor of glutathione synthesis in the lung epithelial cell line A549: transcriptional effect on the GSH rate-limiting enzyme gamma-glutamylcysteine synthetase. Am. J. Respir. Cell Mol. Biol. 17:599-607.

21. Huang, X.Z., Wu, J.F., Spong, S., and Sheppard, D. 1998. The integrin alphavbeta6 is critical for keratinocyte migration on both its known ligand, fibronectin, and on vitronectin. J. Cell Sci. 111:2189-2195.

22. Yokosaki, Y., Monis, H., Chen, J., and Sheppard, D. 1996. Differential effects of the integrins alpha9beta1, alphavbeta3, and alphavbeta 6 on cell proliferative responses to tenascin. Roles of the beta subunit extracellular and cytoplasmic domains. J. Biol. Chem. 271:24144-24150.

23. Sutton, A.B., Canfield, A.E., Shor, S.L., Grant, M.E., and Shor, A.M. 1991. The response of endothelial cells to TGF beta-1 is dependent upon cell shape, proliferative state and the nature of the substratum. J. Cell Sci. 99:777-787.

24. Matthay, M.A., and Wiener-Kronish, J.P. 1990. Intact epithelial barrier function is critical for the resolution of alveolar edema in humans. Am. Rev. Respir. Dis. 142:1250-1257.

25. Modelska, K., et al. 1999. Inhibition of beta-adrenergic-dependent alveolar epithelial clearance by oxidant mechanisms after hemorrhagic shock. Am. J. Physiol. 276:L844-L857.

26. Bunnell, E., and Pacht, E.R. 1993. Oxidized glutathione is increased in the alveolar fluid of patients with the adult respiratory distress syndrome. Am. Rev. Respir. Dis. 148:1174-1178.

27. Das, S.K., and Fanburg, B.L. 1991. TGF-beta 1 produces a "prooxidant" effect on bovine pulmonary artery endothelial cells in culture. Am. J. Physiol. 262:L249-L254.

28. Rao, R.K., Li, L., Baker, R.D., Baker, S.S., and Gupta, A. 2000. Glutathione oxidation and PTPase inhibition by hydrogen peroxide in Caco-2 cell monolayer. Am. J. Physiol. 279:G332-G340.

29. Shenkar, R., Coulson, W.F., and Abraham, E. 1994. Anti-transforming growth factor-beta monoclonal antibodies prevent lung injury in hemorrhaged mice. Am. J. Respir. Cell Mol. Biol. 11:351-357. 